\title{
LARGE-SCALE STRUCTURE AND TERMINATION OF THE HELIOSPHERE
}

\author{
W. M. MACEK \\ Space Research Centre, Polish Academy of Sciences, \\ Bartycka 18 A, PL 00716 Warsaw, Poland; \\ f-mail: macek@obk.waw.pl
}

\begin{abstract}
The question of the boundaries of the heliosphere is considered. The termination heliospheric shock should exist because the solar wind plasma flowing supersonically away from the Sun must make a transition to a subsonic flow. The heliopause is at the outermost extend of the solar wind. Beyond the heliopause lies the (very local) interstellar wind. Intensity of radio emissions at 2 to $3 \mathrm{kHz}$ detected by the Voyager plasma wave instrument in the outer heliosphere can be explained provided that the electron beams generating Langmuir waves exist in the postshock plasma due to secondary shocks in the compressed solar wind beyond the termination shock. The field strengths of Langmuir waves required to generate the second harmonic emissions are $50-100 \mu \mathrm{V} \mathrm{m}^{-1}$. Alternatively, the emissions are generated in the vicinity of the heliopause. The Voyager 1 and 2 are proceeding toward a likely source region for Langmuir wave and these waves may be observed in situ in the near future.
\end{abstract}

\section{Introduction}

Since the 1960's it is known that besides electromagnetic radiation, the Sun also radiates charged particles forming a solar wind. If our solar system moved freely in an empty interstellar space, the solar wind would expand to infinity. Because that is obviously not the case, it is believed that the heliosphere is a finite region of space dominated by the solar wind. Owing to mass conservation, the particle number density of the expanding solar wind should decrease inversely as the square of the distance $r$ from the Sun. The solar wind density already falls below the expected density of the ionized component of the very local inter-stellar medium (VLISM) beyond Saturn's orbit. As a result the heliosphere forms a cavity in the ambient VLISM plasma (Parker, 1963; Axford, 1973; Fahr $6 t$ al., 1986; Suess, 1990). The "nose" of the cavity is located approximately in the solar equatorial plane and is aligned with the apex, i.e., the "head-on" direction of the VLISM velocity. In the down-stream direction of the VLISM the heliosphere is thought to trail into a long comet-like tail. In spite of its asymmetry the cavity is still called the heliosphere; in fact, only near its nose can it be well approximated by a sphere.

As the solar wind expands, the temperature of electrons and ions is reduced and the flow becomes progressively more and more supersonic, reaching the sonic Mach number of about 40 and the Alfv' enic Mach number of $\sim 10$ in the outer heliosphere (Belcher et al., 1993). The inner (termination) heliospheric shock exists because the solar wind must make a transition to a subsonic flow as it runs into the plasma of the VLISM. The heliospheric boundary region is sketched in Figure 1. The heliopause is at the outermost extend of the solar wind. Beyond the heliopause lies the interstellar wind. In this review we only consider some aspects of the physics of the heliospheric boundaries and apologize for incomplete references in this field of research. The interested reader is referred to Proceedings of the Eighth International Solar Wind Conference held in Dana Point, California, USA, 26-30 June 1995, edited by D. Winterhalter $\epsilon t$ al. and published by American Institute of Physics, Woodbury, New York (1996) as well as to Proceedings of the first ISSI Workshop, held in Bern, Switzerland, 6-10 November, 1995, edited by R. von Steiger et al and published in Symer Srirner Revirws, vol. 78, Nos. 1/2 October (1996), see references wherein. 


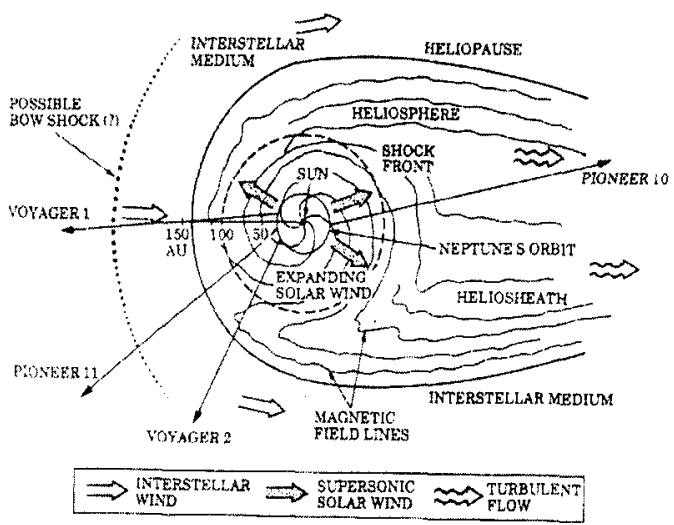

Figure 1. The interaction of the solar wind with the very local inter-stellar medium.

\section{The Structure of the Heliosphere}

The thermodynamic state of the VLISM is still only poorly known. It is even unclear whether or not the VLISM exists in a quasi-stationary near-equilibrium state (Fahr, 1996).

Typical average plasma parameters are listed in Table $1\left(B_{\varphi} \approx B_{\mathrm{r}}\right.$ at $r_{\mathrm{E}}=1 \mathrm{AU}$ and $B \approx B_{\varphi}$ for $r \gg r_{E}$ ). Hence the main contributions to the total pressure $\Pi_{\mathrm{VLISM}}$, in units of $\mathrm{pPa}=10^{-12} \mathrm{~Pa}$, are as follows: the dynamic ram pressure $m n v^{2}=0.105 \mathrm{pPa}$, the magnetic pressure $B^{2} / 2 \mu_{o}=0.064$ $\mathrm{pPa}$ and the thermal pressure of the plasma $p=2 n k T=0.028 \mathrm{pPa}$ ( $m$ is the ion mass and $k$ is the Boltzmann's constant). Since the total solar wind pressure $\Pi_{\mathrm{sw}}=2743 \mathrm{pPa}$ decreases as $r^{-2}$, using pressure balance one obtains the nearly paraboloidal shape of the heliospheric interface (Macek, 1989; Macek et al., 1991a)

$$
\frac{r}{D}=\frac{\delta}{\sin \delta}
$$

where $\delta$ is the angle measured from the Sun-apex line and the distance to the nose of the heliospheric boundary is $D=\left(\Pi_{\mathrm{SW}} / \Pi_{\mathrm{VLISM}}\right)^{1 / 2} r_{\mathrm{E}} \approx 120 \mathrm{AU}$.

TABLE 1. Typical average plasma parameters

\begin{tabular}{llcl}
\hline & VLISM & Solar Wind at $1 \mathrm{AU}$ & and beyond \\
\hline velocity, $v, \mathrm{~km} \mathrm{~s}^{-1}$ & 25 & 450 & $\approx$ const \\
density, $n, \mathrm{~cm}^{-3}$ & $\sim 0.1$ & 8 & $\propto r^{-2}$ \\
temperature, $T, 10^{4} \mathrm{~K}$ & $\sim 1$ & 10 & $\sim$ const \\
magnetic field, $B, \mathrm{nT}$ & $\sim 0.4$ & 5 & $\propto r^{-1}$ \\
\hline
\end{tabular}

This is probably an overestimate, because there are several effects that reduce the distance to the boundaries. First, the interstellar neutral particles enter freely into the heliosphere until they are ionized and then spiral in the interplanetary magnetic field and are carried outward in the solar wind as pick-up ions (Holzer, 1972; Osterbart and Fahr, 1992). Second, the interplanetary magnetic field guides the incoming cosmic ray particles (Fahr et al., 1992; Donohue and Zank, 1993). Both particle populations contribute to the geometry of the heliosphere, acting over vast distances. Third, there is the adverse pressure gradient associated with the curvature of the magnetic field in the region of subsonic flow beyond the shock transition (Holzer, 1972; Axford, 1973).

It is not admittedly clear whether the VLISM is actually supersonic and the heliospheric bow shock exists. After all, the possible bow shock is week, with compression ratio of $1-2$, contrary to the strong termination shock with the expected four-fold density compression. Therefore the models of the boundary region can be divided roughly according to number of interfaces; namely 
we have one-shock and two-shock models. A self-consistent gasdynamic model consisting of two shocks and heliopause (tangential discontinuity) was constructed in a series of papers by Baranov (1990), Baranov and Malama $(1993,1995)$ taking also into account the VLISM neutral particles. These authors solved Boltzmann's equation for the neutral component on the basis of a Monte Carlo approach and found a considerable enhancement in the hydrogen density at the heliopause in the region of the nose. For the discussion of this important feature, called the hydrogen wall, see also (Fahr tt al., 1995) for another approach, and (Pauls et al., 1995) for comparison of one- and two-shock simulations.

With the pass of Ulysses over the pole of the Sun (Phillips et al., 1995), the three-dimensional structure of the solar wind can no longer be ignored. In particular, the Ulysses measurements indicate that the ram pressure of the solar wind increases, by a factor of about 1.5, in going from the ecliptic plane to the solar pole. Simulations of this nonuniform solar wind by Pauls and Zank (1996) show that the solar wind anisotropy results in turbulent flow in the heliotail. This effect is analogous to the production of vorticity in the boundary layer attached to an impulsively moved cylinder in a viscous irrational flow. The temporal variability of the shock has been studied by, e.g., Suess (1993), Ratkiewicz et al. $(1996,1997$ a) and references wherein. Finally, the effects of the interplanetary and/or VLISM magnetic fields on the heliospheric configuration are recently considered by Washimi and Tanaka (1996), Ratkiewicz et al. (1997b), and Nozawa (1997).

In summary, although all of important physical processes has been identified, their relative importance is unknown and the models are not now strongly constrained by the data. The size and shape of the heliospheric cavity is still the subject of conjecture; commonly quoted values of the distance to the termination shock $d$ vary between 70 and $130 \mathrm{AU}$ (see, e.g., Belcher et al., 1993; Zank $\epsilon t$ al., 1994; Steinolfson and Gurnett, 1995; Pauls et al., 1995; Pauls and Zank, 1996).

\section{Radio Emissions from the Heliospheric Boundary}

For several years from 1983 the plasma wave receivers on both Voyagers 1 and 2 spacecraft, located well beyond Saturn's orbit, detected radio emissions at very low frequencies of 2 to $3 \mathrm{kHz}$ (Kurth ct al., 1984, 1987; Kurth, 1990a, b). Since the remote source is observed only at specific phases of the 11-year solar cycle, the radiation was expected to reappear. In fact, some new events have been observed in mid-1989, late 1990 (Kurth and Gurnett, 1991), and the major event was observed from mid-1992 to mid-1993 (Gurnett et al., 1993; Gurnett and Kurth, 1994, 1996; Gurnett, 1995).

The emissions are above the local electron plasma frequency (Kurth, 1990b) and show an upward drift in frequency at a rate of $1-4 \mathrm{kHz} / \mathrm{yr}$ (Kurth et al., 1987, Kurth, 1990a, b; Czechowski and Grz̧edzielski, 1990, 1994). Both main events in 1983-1984 and in 1992-1993 were preceded by intense solar activities produced by interplanetary shocks, which caused the temporary Forbush decreases in cosmic-ray intensities observed in 1982 and 1991, respectively (Gurnett and Kurth, $1995,1996)$. Moreover, in a plane perpendicular to the roll axis of the spacecraft the observed source directions tend to cluster around the apex (or anti-apex) (Gurnett et al., 1993). One expect that the plasma wave receivers will likely provide indicators as well as precursors of the actual crossing of the termination heliospheric shock by the Voyager (Kurth and Gurnett, 1993). Now the Voyager 1 and 2 spacecraft are moving toward he front side of the inner heliospheric shock and the source itself may be reached near the end of this century.

\subsection{GENERATION MECHANISM}

The key to the generation mechanism of the emissions is the spatial structure of the magnetic field. In the outer heliosphere the spiraling interplanetary magnetic field lines become more and more tightly wound (Parker, 1963). So, in the solar equator beyond the planets, the magnetic field lines are within only one degree of being circular, and hence perpendicular to the normal to the heliospheric shock. As is well known from studies of quasi-perpendicular planetary bow shocks, some electrons can be energized streaming along the magnetic field lines (Filbert and Kellogg, 1979; Melrose, 1980).

By analogy with the planetary shocks, (e. g., Gurnett et al., 1989) the beam speed of electrons for the heliospheric shock, $v_{\mathrm{b}}$, is obtained from simple consideration of the field and shock geometry sketched in Figure 1. It can be shown that in order for the electrons to overcome the solar wind convection, the ratio of the beam and solar wind speeds $v_{\mathrm{b}} / v_{\mathrm{SW}}$ near the nose of the shock is 
approximately equal to the distance to this nose, $r=d$, taken in AU (Macek et al., 1991a). The velocity of the solar wind flow is only a small fraction of the velocity of light, $v_{\mathrm{SW}}=0.0015 \mathrm{c}$. Taking, e.g., $d=100 \mathrm{AU}$ one finds $v_{\mathrm{b}}=0.15 \mathrm{c}$, corresponding to an electron energy of $\sim 6 \mathrm{keV}$ near the nose of the heliospheric shock. Very large beam speeds are also found on the whole meridian passing through the nose except near the solar poles, because near the poles the field lines are such that the shock is no longer quasi-perpendicular.

Following Kurth et al. (1984), Macek et al. (1991a, b) first presented a simple model for the generation mechanism of the emission due to nonlinear interaction between Langmuir waves generated by the electron beam near the heliospheric shock and considered kinematic constraints on the emission processes involving Langmuir waves. In this way, they estimated the wave amplitude expected to be measured by the plasma wave instrument in the heliospheric foreshock. In the limiting case of saturation of these emission processes the minimum value of the wave electric field of $3-30 \mu \mathrm{V} \mathrm{m}^{-1}$ was estimated, and more realistic values of $100-200 \mu \mathrm{V} \mathrm{m}^{-1}$ were calculated by Macek (1994).

Surely, if the inner heliospheric shock is a source emitting at the second harmonic of the plasma frequency, $2 f_{\mathrm{p}}=2-3 \mathrm{kHz}$, it is difficult to obtain so high electron density $\left(n_{\mathrm{e}}=(1-3) \times 10^{-2} \mathrm{~cm}^{-3}\right)$ required in the source in the outer heliosphere, say, at the heliospheric distance $r \sim 10^{2} \mathrm{AU}$. Since $n_{\mathrm{e}}$ in the solar wind varies as $r^{-2}$, one would need to have at $1 \mathrm{AU} n_{\mathrm{e}} \sim 10^{2} \mathrm{~cm}^{-3}$. This difficulty could be much alleviated if the electron beams generating Langmuir waves existed also in the postshock plasma due to secondary shocks in the compressed solar wind beyond the termination shock (Grzedzielski, 1993; Macek, 1996), as sketched in Figure 2. The field strength of Langmuir waves in the secondary foreshocks was also calculated to be of $50-100 \mu \mathrm{V} \mathrm{m}^{-1}$ (Macek et al., 1995; Macek, 1996 ). Alternatively, the emissions are generated in the vicinity of the heliopause, as first suggested by Fahr et al. (1986), possibly in the hydrogen wall (Gurnett et al., 1993). However, in the latter case the mechanism of the emissions remains unknown.

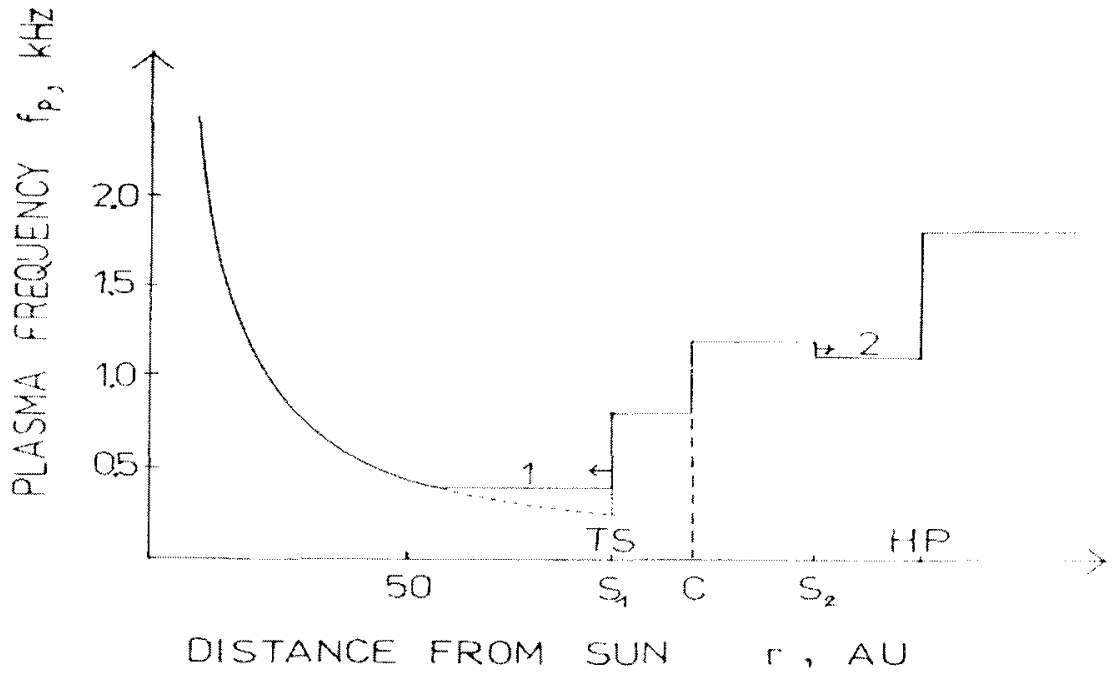

Figure 2. The plasma frequency $f_{\mathrm{p}}$ profiles of the heliospheric shocks. The termination shock (TS) is split into the primary $\left(S_{1}\right)$ and secondary $\left(S_{2}\right)$ shocks, separated by a contact discontinuity $(C)$. The respective shocks are denoted by 1 and 2 . The secondary shock is propagating in the shocked solar wind plasma toward the heliopause $(H P)$, this figure is taken from (Macek, 1996).

\section{Discussion}

Voyager 1 is traveling with a velocity of $3.5 \mathrm{AU} / \mathrm{yr}$ at $\sim 35^{\circ}$ north of the ecliptic and is expected to be at $r \sim 70 \mathrm{AU}$ in 1998 and $r \sim 100 \mathrm{AU}$ in 2006. After Neptune, Voyager 2 is traveling $\sim 45^{\circ}$ south of the ecliptic. It is fortunate that the beam velocity and, consequently, the measured 
wave amplitude and the flux density are less sensitive to the latitudinal than to the longitudinal variations from the actual nose of the shock. Hence both these spacecraft may be approaching the regions of the heliospheric shock where $v_{\mathrm{b}}$ is calculated to be large, and so a likely source region for Langmuir waves. However, Voyager 1 and 2 will not have enough power to operate much beyond the year 2015, when they will be at $r \sim 130 \mathrm{AU}$ and $r \sim 105 \mathrm{AU}$, respectively. If the termination shock is located at $r \leq 100 \mathrm{AU}$ or so, these Langmuir waves may, in principle, be observed in situ in the near future.

Until now, with the Voyager spacecraft still located far from the Langmuir wave source region, only escaping electromagnetic radiation from a remote source may have been detected (Kurth $e t$ al., 1984, 1987; Gurnett et al., 1993), with maximum flux densities observed in late 1983 and 1992 only a factor of two above the instrumental threshold.

\section{Conclusions}

The termination heliospheric shock should exist because the solar wind plasma flowing highly supersonically away from the Sun must make a transition to a subsonic flow. The heliopause is at the outermost extend of the solar wind. Beyond the heliopause lies the (very local) interstellar wind. The size of the heliospheric cavity is still the subject of conjecture; commonly quoted values of the distance to the nose of the terminal shock vary between 70 and $130 \mathrm{AU}$.

The two strong heliospheric emission events at 2 to $3 \mathrm{kHz}$ have been observed by Voyagers in 1983-1984 and 1992-1993. The suggested mechanism of the emission is due to nonlinear interaction between Langmuir waves generated by electron beams in the heliospheric foreshock at multiplies of the local plasma frequency.

Comparison of the discussed results with electromagnetic radiation coming from upstream of the Earth's bow shock shows that the model adequately explains the generation of plasma waves at planetary shocks. Intensity of radio emissions at 2 to $3 \mathrm{kHz}$ can also be explained provided that the electron beams generating Langmuir waves exist in the postshock plasma due to secondary shocks in the compressed solar wind beyond the termination shock. Admittedly, this is one possible explanation of the observations. The field strength of Langmuir waves required to generate the second harmonic emissions are approximately of $100-200 \mu \mathrm{V} \mathrm{m}$ m $^{-1}$ for the primary and $50-100 \mu \mathrm{V} \mathrm{m}^{-1}$ for the secondary foreshocks. However, only in the secondary foreshock the expected density is consistent with the observed frequency.

The Voyager 1 and 2 are proceeding toward the boundary of the heliosphere, a likely source region for Langmuir waves, and these waves may be observed in situ in the near future.

\section{Acknowledgments}

This work was supported by the Scientific Research Committee Grant 2P03C 01210.

\section{References}

Axford, W. I. (1973) Interaction of the Interstellar Medium With the Solar Wind, Space Sci. Rev., 14, 582-590.

Baranov, V. B. (1990) Gasdynamics of the Solar Wind Interaction With the Interstellar Medium, Space Sci. Rev., 52, $89-120$.

Baranov, V. B. and Malama, Yu. G. (1993) Model of the Solar Wind Interaction With the Local Interstellar Medium: Numerical Solution of Self-Consistent Problem, J. Geophys. Res., 98, 15157-15163.

Baranov, V. B and Malama, Y. G. (1995) Effect of Local Interstellar Medium Hydrogen Fractional Ionization on the Distant Solar Wind and Interface Region, J. Geophys. Res., 100, 14755-14761.

Belcher, J. W., Lazarus, A. J., McNutt, R. L. Jr. and Gordon, G. S. Jr. (1993) Solar Wind Conditions in the Outer Heliosphere and the Distance to the Termination Shock, J. Geophys. Res., 98, 15177-15183.

Czechowski, A. and Grzsedzielski, S. (1990) Frequency Drift of 3-kHz Interplanetary Radio Emissions: Evidence of Fermi Accelerated Trapped Radiation in a Small Heliosphere?, Nature, 344, 640-641.

Czechowski, A. and S. Grzedzielski, S. (1994) Can a Charge-Exchange Induced Density Rise at the Heliopause Explain the Frequency Drift of the $3 \mathrm{kHz}$ Voyager Signal?, Geophys. Res. Lett., 21, 2777-2780.

Donohue, D. J. and Zank, G. P. (1993) Steady State and Dynamical Structure of a Cosmic-Ray-Modified Termination Shock, J. Geophys. Res., 98, 19005-19025.

Fahr, H. J. (1996) The Interstellar Gas Flow Through the Heliospheric Interface Region, Space Sci. Rev., 78, $199-212$.

Fahr, H. J., Neutsch, W., Grzedzielski, S., Macek, W. M. and Ratkiewicz-Landowska, R. (1986) Plasma Transport Across the Heliopause, Space Sci. Rev., 43, 329-381.

Fahr, H. J., Fichtner, H. and Grzedzielski, S. (1992) The Influence of the Anomalous Cosmic-Ray Component on the Dynamics of the Solar Wind, Solar Phys., 137, 355-383. 
Fahr, H. J., Osterbart, R. and Rucinski, D. (1995) Modulation of the Interstellar Oxygen-to-Hydrogen Ratio by the Heliospheric Interface Plasma, Astron. Astrophys., 294, 587-600.

Filbert, P. C. and Kellogg, P. J. (1979) Electrostatic Noise at the Plasma Frequency Beyond the Earth's Bow Shock, J. Geophys. Res., 84, 1369-1381.

Grzedzielski, S. (1993) in W. M. Macek (ed.) Emission of 2-3 kHz Band From the Shocked Solar Wind, The VLF Emissions in the Heliosphere, Proc. of a Topical Workshop, Warsaw, 8-12 June 1992, International Heliospheric Study Newsletter, 7, April 1993, pp. 51-55.

Gurnett, D. A. (1995) Heliospheric Radio Emissions, Space Sci. Rev., 72, 243-254.

Gurnett, D. A. and Kurth, W. S. (1994) Evidence that Jupiter is not the Source of the 2-3 kHz Heliospheric Radiation, Geophys. Res. Lett., 21, 1571-1574.

Gurnett, D. A. and Kurth, W. S. (1995) Heliospheric 2-3 kHz Radio Emissions and their Relationship to Large Forbush Decreases, Adv. Space Res., 16, (9)279-290.

Gurnett, D. A. and Kurth, W. S. (1996) Radio Emissions from the Outer Heliosphere, Space Sci. Rev., 78, 53-66.

Gurnett, D. A., Kurth, W. S., Poynter, R. J., Granroth, L. J., Cairns, I. H., Macek, W. M., Moses, S. L., Coroniti, F. V., Kennel, C. F. and Barbosa, D. D. (1989) First Plasma Wave Observations at Neptune, Science, 246, 1494-1498.

Gurnett, D. A., Kurth, W. S., Allendorf, S. C. and Poynter, R. J. (1993) Radio Emission from the Heliopause Triggered by an Interplanetary Shock, Science, 262, 199-203.

Folzer, T. E. (1972) Interaction of the Solar Wind with the Neutral Component of the Interstellar Gas, J. Geophys. Res., 77, 5407-5431.

Kurth, W. S. (1990a) Sounding a Small Heliosphere, Nature, 344, 586-587.

Kurth, W. S. (1990b) in S. Grzedzielski and D. E. Page (eds.), Radio Noise in the Heliospheric Cavity, Physics of the Outer Heliosphere, COSPAR Colloquia, vol. 1, Pergamon, New York, pp. 267-275.

Kurth, W. S. and Gurnett, D. A. (1991) New Observations of the Low-Frequency Interplanetary Radio Emissions, Geophys. Res. Lett., 18, 1801-1804.

Kurth, W. S. and Gurnett, D. A. (1993) Plasma Waves as Indicators of the Termination Shock, J. Geophys. Res., 98, 15129-15136.

Kurth, W. S., Gurnett, D. A., Scarf, F. L. and Poynter, R. L. (1984) Detection of a Radio Emission at $3 \mathrm{kHz}$ in the Outer Heliosphere, Nature, 312, 27-31.

Kurth, W. S., Gurnett, D. A., Scarf, F. L. and Poynter, R. L. (1987) Long-Period Dynamic Spectrograms of LowFrequency Interplanetary Radio Emissions, Geophys. Res. Lett., 14, 49-52.

Macek, W. M. (1989) Reconnection at the Heliopause, Adv. Space Res., 9, (4)257-261.

Macek, W. M. (1994) Mechanism of Low-Frequency Radio Emissions in the Heliosphere, Geophys. Res. Lett., 21, 249-252.

Macek, W. M. (1996) Emission Mechanism for Low-Frequency Radiation in the Outer Heliosphere, Space Sci. Rev., 76, 231-250.

Macek, W. M, Cairns, I. H. Kurth, W. S. and Gurnett, D. A. (1991a) Plasma Waves Generation Near the Inner Heliospheric Shock, Geophys. Res. Lett., 18, 357-360.

Macek, W. M., Cairns, I. H., Kurth, W. S. and Gurnett, D. A. (1991b) Low-Frequency Radio Emissions in the Outer Heliosphere: Constraints on Emission Processes, J. Geophys. Res., 96, 3801-3806.

Macek, W. M., Czechowski, A. and Grzedzielski, S. (1995) Mechanism for Generation of Radio Emissions from the Planetary and Heliospheric Foreshocks, Adv. Space Res., 15, 467-474.

Melrose, D. B. (1980) Plasma Astrophysics, Gordon and Breach, New York.

Nozawa, S. (1997) Effect of Magnetic Field in the 3-Dimensional Heliosphere, Highlights in Astronomy, this issue

Osterbart, R. and Fahr, H. J. (1992) A Boltzmann-Kinetic Approach to Describe the Entrance of Neutral Interstellar Hydrogen into the Heliosphere, Astron. Astrophys., 264, 260-269.

Parker, E. N. (1963) Interplanetary Dynamical Processes, Interscience, New York.

Pauls, H. L. and Zank, G. P. (1996) Interaction of a Nonuniform Solar Wind with the Local Interstellar Medium, $J$. Geophys. Res., 101, 17081-17092.

Pauls, H. L., Zank, G. P. and Williams, L. L. (1995) Interaction of the Solar Wind with the Local Interstellar Medium, J. Geophys, Res., 100, 21595-21604.

Phillips, J. L., Bame, S. J., Feldman, W. C., Goldstein, B. E., Gosling, J. T., Hammond, C. M., McComas, D. J., Neugebauer, M., Scime, E. E. and Suess, S. T. (1995) Ulysses Solar Wind Plasma Observations at High Southerly Latitudes, Science, 268, 1030-1033.

Ratkiewicz, R., Barnes A., Molvik, G. A., Spreiter, J. R. and Stahara S. S. (1996) Heliospheric Termination Shock Motion due to Fluctuations in the Solar Wind Upstream Condition: Spherically Symmetric Model, J. Geophys. Res., 101, 27483-27497.

Ratkiewicz, R., Barnes A. and Spreiter, J. R. (1997a) Heliospheric Termination Shock Motion in Response to LISM Variation: Spherically Symmetric Model, Geophys. Res. Lett., 24, 1659-1662.

Ratkiewicz, R., Barnes, A., Molvik, G. A., Spreiter, J. R., Stahara, S. S., Vinokur, M. and Venkateswaran, S. (1997b) Effect of the Local Interstellar Magnetic Field on the Heliospheric Configuration, Eos Trans. AGU, 77, (46) F575, AGU 1996 Fall Meeting, 15-19 December, 1996, San Francisco, USA;

Effect of Varying Strength and Orientation of Local Interstellar Magnetic Field on Configuration of Exterior Heliosphere: 3D MHD Simulations, Astron. Astrophys., submitted.

Steinolfson, R. S. and Gurnett, D. A. (1995) Distances to the Termination Shock and Heliopause from a Simulation Analysis of the 1992-93 Heliospheric Radio Emission Event, Geophys. Res. Lett., 22, 651-654.

Suess, S. T. (1990) The Heliopause, Rev. Geophys., 28, 97-115.

Suess, S. T. (1993) Temporal Variations in the Termination Shock Distance, J. Geophys. Res., 98, 15147-15155.

Washimi, H. and Tanaka, T. (1996) 3-D Magnetic Field and Current System in the Heliosphere, Space Sci. Rev., 78, $85-94$.

Zank, G. P., Cairns, I. H., Donohue, D. J. and Matthaeus, W. H. (1994), Radio Emissions and the Heliospheric Termination Shock, J. Geophys. Res., 99, 14729-14735. 